\title{
La professionnalisation policière en situation coloniale : détour conceptuel et explorations historiographiques
}

Emmanuel Blanchard, Quentin Deluermoz et Joël Glasman

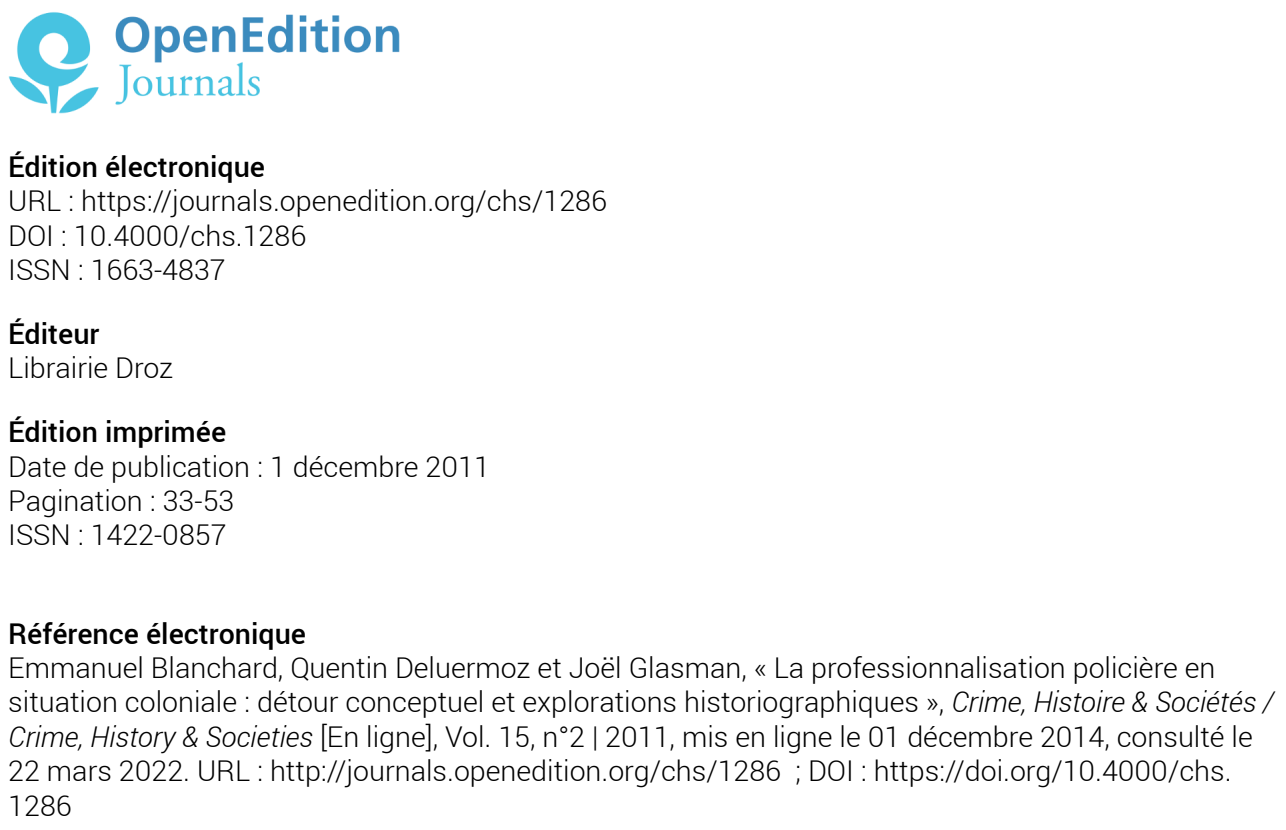




\title{
La professionnalisation policière en situation coloniale:
}

\author{
détour conceptuel et explorations historiographiques
}

\author{
Emmanuel Blanchard', \\ Quentin Delvermoz ${ }^{2}$, Joël Glasman ${ }^{3}$
}

\begin{abstract}
The historiography of European and North American police forces during the 19th and 20th centuries has placed strong emphasis on the move towards specialisation and professionalization, which would have helped to contain the use of violence by policing agencies. These developments were paralleled by the establishment of colonial police forces. In these dependent territories, hybrid forms of police organisations, partly civilian partly militarised, remained more dominant than in the imperial centres. The connection between these territories facilitated the circulation of practices and personnel as well as shared points of reference. Therefore the study of the structures of these policing agencies, their use of lethal violence, the communication between new and traditional agents, and the relationship between civilian populations and colonial policemen, call for a more nuanced appreciation of the idea of police professionalization as the driving force in the civilising process of public order maintenance.
\end{abstract}

1 Emmanuel Blanchard, maître de conférences à l'Université de Versailles Saint-Quentin en Yvelines et chercheur au CESDIP (UMR 8183, CNRS-ministère de la Justice-UVSQ). Sa thèse a été publiée en octobre 2011 sous le titre La police parisienne et les Algériens, Paris, Nouveau Monde éd. Parmi ses autres publications: «Quand les forces de police défient le palais Bourbon (13 mars 1958). Les policiers manifestants, l'arène parlementaire et la transition de régime », Genèses. Sciences sociales et histoire, 2011, 83, pp. 55-73; «L'encadrement des Algériens de Paris (1944-1954), entre contraintes juridiques et arbitraire policier», Crime, Histoire \& sociétés/Crime, History and Societies, 2007, 1, 1, pp. 5-25; «Police judiciaire et pratiques d'exception pendant la guerre d'Algérie», Vingtième Siècle. Revue d'histoire, avril-juin 2006, 90, pp. 61-72.

2 Quentin Deluermoz, maître de conférences en histoire contemporaine à l'Université de Paris 13 (CRESC, EA 2356). Sa thèse sera publiée à l'automne 2011 sous le titre Policiers dans la ville. La construction d'un ordre public parisien (1854-1914), Paris, Publications de la Sorbonne. Parmi ses autres publications: «Présences d'État, une interrelation police-société à Paris (1854-1880)», Annales, HSS, 2009, 2, pp. 435-460; «La police en tenue à Paris dans la seconde moitié du $\mathrm{XIX}^{\mathrm{e}}$ siècle: du modèle londonien au modèle parisien?», Revue d'histoire des sciences humaines, déc. 2008, 19, pp. 75-90. Il a également dirigé un numéro spécial de la revue Vingtième Siècle. Revue d'histoire consacré à «Norbert Elias et le $\mathrm{XX}^{\mathrm{e}}$ siècle: le processus de civilisation à l'épreuve» (avril 2010, 106).

3 Joël Glasman, assistant de recherche, département d'études africaines, Humboldt Universität Berlin. Il a soutenu une thèse sur Les Corps habillés. Genèse des métiers de police au Togo (1885-1963), thèse de l'université de Leipzig et de l'université Paris 7-Denis-Diderot, 2011. Il est notamment l'auteur de: «Penser les intermédiaires coloniaux. Note sur les dossiers de carrière de la police du Togo », History in Africa, 2010, 37, pp. 51-81; « La troupe de police du Togo allemand. L'ordre colonial entre discours et pratiques (1885-1914)», in Chatriot A., Gosewinkel D., Dieter (Eds), Koloniale Politik und Praktiken. Deutschlands und Frankreichs 1880-1962, Steiner Verlag, Wiesbaden 2010, pp. 29-50. 


\begin{abstract}
L'historiographie des polices européennes et d'Amérique du Nord aux $X I X^{e}$ et $X X^{e}$ siècles a beaucoup mis l'accent sur un mouvement de spécialisation et de professionnalisation favorisant la contention de la violence des forces de l'ordre. Ces évolutions furent concomitantes de l'émergence de polices coloniales. Dans ces espaces dominés, le caractère hybride des forces civiles et militaires est resté plus prégnant que dans les métropoles impériales. Surtout, les connections entre ces espaces se sont traduites par des circulations de pratiques, d'acteurs et de référentiels. Ainsi, l'étude du format des forces armées, de l'usage de formes létales de violence, de l'articulation entre unités nouvelles et vernaculaires ou des relations entre populations et agents en situation coloniale invite à nuancer le topos d'une professionnalisation policière vectrice d'une civilisation du maintien de l'ordre.
\end{abstract}

L e concept de professionnalisation est devenu central dans l'historiographie de la police. Des grandes synthèses sur l'histoire policière aux colloques internationaux, en passant par les monographies sur l'histoire policière, il est largement utilisé dans les ouvrages récents sur le sujet ${ }^{4}$. Pour incontournable qu'il semble être, ce concept ne va pourtant pas sans poser question. Les historiens et les historiennes qui l'utilisent sont les premiers à reconnaître que la professionnalisation des polices européennes ne fut pas un processus linéaire et uniforme. Tous reconnaissent les heurts, les tâtonnements, les détours, les reculs qui ont caractérisé les chemins souvent incertains et parfois chaotiques de la professionnalisation policière.

L'idée défendue ici est que le détour par la situation coloniale peut contribuer à enrichir la problématique de la «professionnalisation» policière telle qu'elle est envisagée habituellement. Sans récuser en bloc ce concept, l'objectif est d'ouvrir à nouveau frais la réflexion sur un processus complexe, au regard d'un matériel empirique longtemps négligé et récemment redécouvert: celui issu des recherches sur la genèse et l'action des forces de l'ordre dans les empires coloniaux des $\mathrm{XIX}^{\mathrm{e}}$ et $\mathrm{XX}^{\mathrm{e}}$ siècles ${ }^{5}$. Il ne s'agit donc pas, loin s'en faut, d'apporter des réponses définitives à une question dont l'historiographie, celle des polices coloniales, est encore largement en friche ${ }^{6}$, mais bien de proposer quelques pistes de réflexion.

Comment, dès lors, comprendre ce qui est souvent lu, malgré la différence des temporalités et des intensités, comme des évolutions ou des «transferts» s'apparentant à une forme de «professionnalisation» en situation coloniale? Que peut-nous apprendre en retour la trajectoire coloniale sur un « moment historiographique ${ }^{7}$ largement cadré par les espaces métropolitains? Tester les apports heuristiques d'un décloisonnement géographique nécessite d'abord de retracer brièvement les usages de la catégorie de «professionnalisation» dans l'étude des polices européennes et coloniales (I). Pour questionner ce concept, il est ainsi nécessaire de revenir sur

4 Berlière (1996); Berlière, Denys, Kalifa, Milliot (2008); Denys (2002).

5 Une partie des analyses de ce texte et des articles du dossier trouvent leur origine dans les travaux et réflexions présentés dans le cadre du séminaire «Polices et policing en situation coloniale» organisé par le GERN. Des journées d'étude se sont tenues à Milton Keynes (sept. 2009, dir. G. Sinclair), Paris (nov. 2009, dir. E. Blanchard), Leiden (sept. 2010, dir. M. Bloembergen, M. De Koster) et Porto (avril 2011, dir. C. O'Reilly).

6 Pour un premier bilan, centré sur le continent africain, Glasman (2010).

7 Milliot (2007). 
l'émergence de ces dernières, en particulier la «greffe» d'organisations d'inspiration métropolitaine sur les institutions vernaculaires (II). La notion de «consentement» à l'action des forces de l'ordre doit ainsi être questionnée à l'aune d'une séparation armée-police à peine esquissée en situation coloniale (III).

\section{PROFESSIONNALISATION ET CIVILISATION DES MOEURS POLICIĖRES}

Dans l'histoire des structurations sociales des XVIII ${ }^{\mathrm{e}}-\mathrm{XX}^{\mathrm{e}}$ siècles européens, la notion de professionnalisation est courante et semble renvoyer à un ensemble assez bien fixé d'éléments identifiant des processus de spécialisation et d'institutionnalisation croissants: mise en place de procédures de recrutement, spécialisation des savoirs, établissement de structures de formation, amélioration de la paie en vue de limiter la corruption, expression croissante de l'exigence disciplinaire... Mobilisée avec évidence, la notion n'est cependant en rien monolithique. Elle est largement débattue par les sociologues, partagés entre les définitions fonctionnalistes, plus attachées à l'établissement d'un monopole institué sur une activité, et une lecture interactionniste davantage axée sur la sédimentation sociale des identités professionnelles ${ }^{8}$. La dimension processuelle a également été mise en avant, notamment dans les travaux d'Andrew Abbott ${ }^{9}$. Sont alors soulignés le rôle de la fin du XIX ${ }^{\mathrm{e}}$ occidental marqué par l'extension du salariat, la rationalisation des processus de travail, l'alphabétisation ou l'affirmation de l'État-nation, même si d'autres travaux rappellent le poids des structures de plus longue durée ${ }^{10}$. La notion fait bien débat, tout en semblant toucher au cœur de transformations sociales de grande ampleur, ce qui explique d'ailleurs qu'elle fasse souvent l'objet, chez les historiens, d'un usage relâché qui contourne ces interrogations.

La notion de professionnalisation pose en outre un certain nombre de problèmes. Elle propose d'abord un cadre quelque peu téléologique qui fait tendre les mutations relevées au cours du $\mathrm{XIX}^{\mathrm{e}}$ siècle vers un aboutissement prédéterminé. De nombreux travaux sur les «fonctionnaires intermédiaires » montrent pourtant le rôle décisif des années 1830-1840, considérées en elles-mêmes ${ }^{11}$. Le concept recouvre souvent un jugement de valeur positif, même implicite. Dans bien des cas, la professionnalisation apparaît comme le révélateur des conditions sociales qui la rendent possible, et qui sont elles-mêmes simplifiées dans des catégories très générales: la professionnalisation est souvent décrite comme l'expression d'une «modernisation» en cours des sociétés.

Le phénomène est particulièrement net pour la professionnalisation des forces de police, à travers laquelle se jouent tout à la fois le rapport à l'État, à la norme, à la violence ou au quotidien. Les récits de la modernisation policière, à vrai dire, sont doubles. Une version, plus proche du marxisme ou d'une histoire sociale des mouvements ouvriers, a vu dans la structuration des forces de police la mise en place

\footnotetext{
8 Champy (2009).

9 Abbott (1988). Selon Abbott, les années 1860-1880 voient lentement émerger les conditions sociales, culturelles et politiques du champ professionnel contemporain.

10 Karpik (1995).

11 Le Bihan (2008).
} 
d'un outil de gouvernement au service des groupes possédants. Une autre, plus libérale, a salué à travers ce phénomène la progressive extension des lois, des libertés et de la démocratisation ${ }^{12}$. Les analyses qui insistent sur le mouvement de «professionnalisation » sont en général plus proches de cette dernière perspective.

Le récit trace alors le parcours d'une police qui se dégage de son origine militaire (pour les polices européennes continentales), devient mieux contrôlée, plus disciplinée, plus douce et participe ainsi à un plus vaste processus de «pacification des mœurs », suivant une lecture trop rapide des travaux de Norbert Elias ${ }^{13}$. Rappelons que pour ce dernier, le «processus de civilisation » désignait un mouvement de très longue durée $\left(\mathrm{XIII}^{\mathrm{e}}-\mathrm{XX}{ }^{\mathrm{e}}\right.$ siècles) articulant ensemble l'affirmation de l'État «moderne», l'accroissement des interdépendances et le développement d'une nouvelle économie psychique fondée sur une plus grande retenue des comportement. Pour Elias, ce processus n'est cependant pas affecté d'une connotation positive, il est capable d'inversion et n'a rien de garanti ${ }^{14}$.

Mais ces précisions ne sont souvent pas prises en compte dans les approches comparatives des polices occidentales. Hsi-Huey Liang, en étudiant les évolutions à partir d'une série de critères (loi, recrutement, etc.), montrait ainsi en 1992 une convergence professionnelle et démocratique des polices occidentales (RoyaumeUni, France, Allemagne, USA) au cours des $\mathrm{XIX}^{\mathrm{e}}-\mathrm{XX}^{\mathrm{e}}$ siècles ${ }^{15}$. D'autres travaux ont tâché de concilier ce mouvement avec une meilleure appréhension des profils nationaux, dégageant des «voies plurielles de la modernisation» en matière de police $^{16}$. Cela permettait notamment de souligner les deux «exceptions »: la britannique, par l'achèvement du processus, l'allemande, par le poids persistant du modèle militaire. Percent ici des lectures consacrées des différences nationales (notamment les fameux «exceptionnalisme» anglais et «Sonderweg» allemand) indexées à un modèle de référence, pluriel ou non, qui reste évolutionniste.

Naturellement, les transformations des polices coloniales ont été analysées, dans nombre de travaux européens, à l'aune de cette perspective. La situation est particulièrement nette pour l'appréciation de l'empire anglais, où la tradition «whig», renforcée par l'association entre le «modèle londonien» et la perfection politique britannique, a connu une longue vigueur. Pour Charles Jeffries, auteur d'un célèbre The colonial police paru en 1952, les polices de l'empire britannique suivraient ainsi trois phases: la première relève d'un bricolage maladroit, la seconde prend des traits semi-militaires sous l'impulsion du «modèle» de la Royal Irish Constabulary, la dernière évolue vers une police moins armée, plus «civile» et plus «britannique»-qui correspond au moment de la rédaction de l'ouvrage ${ }^{17}$. Jeffries insiste particulièrement sur le cas de Ceylan qui lui paraît exemplaire: en 1844, J.S. Colepeper, sergent dans la Metropolitan police de Londres, devenu superintendant à Ceylan, réorganise la force de police de Colombo selon les lignes de la Metropolitan police. Mais, inadap-

12 Pour un aperçu de ces deux récits dans différents espaces européens, voir Emsley, Reinke, Lévy (1994).

13 Collectif (2010).

14 Elias (1939).

15 Liang (1992).

16 Knöbl (1998).

17 Jeffries (1952). 
tée à la population, elle suscite des moqueries et se caractérise par une grande inefficacité. T. Thompson, de la Royal Irish Constabulary, puis W.I. Macartney assurent à partir de 1848 le passage à un modèle plus militaire, «à l'irlandaise ${ }^{18}$, qui s'avère plus convaincant, quoiqu'également peu efficace dans la lutte contre la criminalité. Les forces de police conservent cet aspect jusqu'à la guerre et il faut attendre l'arrivée de Herbert Dowbigging, inspecteur général de Ceylan de 1913 à 1937, pour voir les mutations finales. Sous son égide se met en place la police de 1948, «efficace, disciplinée et loyale», «à l'anglaise». Sa réussite est telle qu'elle est même devenue un modèle pour le reste de l'empire britannique (Zanzibar, Chypre, Gambie, Niger, Palestine, Kenya). Dans cet ouvrage, rédigé par un ancien secrétaire d'État aux colonies, le poids de l'idéologie coloniale est évident. Mais, même chez les historiens, la notion reste souvent associée à cette perspective d'ensemble. Il fallut attendre les premiers travaux issus des aires anciennement colonisées ou ouverts aux représentations et capacités d'action des «subalternes » pour que l'écriture de l'histoire des polices en situation coloniale s'affranchisse de ces perspectives: les travaux successifs sur l'Inde ou le Nigéria sont emblématiques de ces renouvellements historiographiques $^{19}$.

Ces constats ne doivent pas pour autant amener à négliger les évidentes mutations qui affectent les espaces européens et qui servent de références à la notion de professionnalisation. En France, par exemple, les travaux sur l'histoire de la police ou de la gendarmerie ont bien montré cette instauration d'un recrutement plus sourcilleux, d'une formation régulière, d'un contrôle hiérarchique accru mais aussi l'intégration sociale autant que symbolique de ces forces, au point que la présence massive de leurs agents, difficilement imaginable au début du XIX ${ }^{\mathrm{e}}$ siècle, apparaît évidente à la Belle Époque ${ }^{20}$.

Ces travaux ont cependant aussi amorcé une discussion plus ferme avec la notion et ses sous-entendus. Les travaux sur le XVIII siècle ont ainsi défini une «professionnalisation» identifiant l'autonomisation des savoirs, la meilleure définition du mot «police» et une spécialisation de groupes reliés plus directement à l'État. Le choix du terme peut surprendre mais l'intention, évidente, est précisément de rompre avec des lectures trop téléologiques pour insister sur les configurations successives à l'œuvre ${ }^{21}$. Parce qu'elle détache les groupes policiers des univers sociaux dans lesquels ils étaient immergés, la militarisation, dans cette acception, peut alors être considérée comme un élément de cette professionnalisation ${ }^{22}$. Les travaux sur le $\mathrm{XIX}^{\mathrm{e}}$ siècle ont proposé de semblables déplacements ${ }^{23}$. Ils ont notamment dégagé l'ambiguïté du processus: l'examen du recours à la gendarmerie en cas d'émeute à la fin du XIX ${ }^{\mathrm{e}}$ suggère par exemple qu'en rendant l'action policière plus cohérente, la professionnalisation rend son usage de la force plus efficace également; ce mouvement, de plus, n'empêche pas ensuite le recours à la violence, selon des cir-

\footnotetext{
18 Sur ce modèle, voir Sinclair (2008).

19 Arnold (1986); Gooptu (2004); Ahire (1991).

20 Berlière (1990); Deluermoz (2009); Houte (2010).

21 Milliot (2008, p. 33).

22 Denys (2002).

23 Lignereux (2008); Houte (2010); Deluermoz (2006). Sur le rapport à l'autorité, Collectif (2008).
} 
constances qui ne sont pas forcément conjoncturelles; enfin, il accompagne une mutation qui est aussi celle des relations de pouvoir ${ }^{24}$. Certaines recherches ont en outre fait de la «modernisation» un objet de l'analyse plus qu'une catégorie en soi : le discours sur la «modernité», thème intense de l'imaginaire du temps, est aussi un des modes de construction de la légitimité policière. En devenant plus fermement associés à l'idée de «civilisation » à la fin du siècle, les gardiens de la paix parisiens, parmi d'autres, s'inscrivent ainsi dans une logique symbolique opposant cette civilisation aux forces de barbarie, justifiant ou tendant à justifier la violence à l'égard de ces derniers ${ }^{25}$.

Les travaux sur le $\mathrm{XX}^{\mathrm{e}}$ siècle enfin, en histoire ou en sociologie, ont nourri ces réagencements en portant la réflexion au cœur d'une police déjà professionnelle dans laquelle l'usage de la force, pas forcément en déclin, fait l'objet d'apprentis$\operatorname{sage}^{26}$. Le débat sur la définition de l'action policière, partagé entre usage de la contrainte et nécessité du consentement, en est une illustration. Pour Egon Bittner, en dépit de l'apparent adoucissement des relations, «le rôle de la police se définit comme un mécanisme de distribution d'une force coercitive non négociable, mis au service d'une compréhension intuitive des exigences d'une situation (...) Le droit de la police à l'usage de la force ne comprend essentiellement aucune restriction $\gg^{27}$. Chez d'autres auteurs, ce mouvement de pacification du maintien de l'ordre n'en est pas moins manifeste dans les sociétés occidentales. Il suppose notamment que l'autocontrainte et la discipline soient telles qu'elles puissent se substituer à la démonstration d'une force pure visant à refonder un pacte politique mis à mal par la violence de la contestation: c'est ainsi que pour Jean-Paul Brodeur «la police ne peut opérer que dans le contexte d'une pacification antécédente. Quand le contrat de nonviolence est collectivement rompu, la police est battue; il faut lui substituer un autre corps violent, l'armée, des milices ou des mercenaires appartenant au secteur privé $»^{28}$. Une telle situation, indique le même auteur, n'empêche pas les heurts et n'est jamais pleinement acquise.

Ainsi, de même que l'extension de la «société civile» appuie la démocratisation des sociétés contemporaines, mais ne s'y réduit pas, voire peut s'y opposer ${ }^{29}$, il semble que la professionnalisation policière accompagne dans certains espaces l'intégration de l'État-nation et les nouveaux seuils de sensibilité à la violence, mais qu'elle ne s'y limite pas et peut entraîner au contraire des inversions, progressives ou brutales. Les colonies n'apparaissent alors plus comme des récepteurs passifs de processus venus d'Europe, mais au contraire comme des terres où des pratiques furent testées, ou au moins utilisées ${ }^{30}$, avant que la circulation des hommes et des savoirs ne les acclimatent sous d'autres latitudes. Il faut toutefois, pour bien saisir

\footnotetext{
$24 \quad$ Collectif (2008).

25 Deluermoz (2006).

26 Dewerpe (2006); Jobard (2002); Pruvost (2007); Moreau de Bellaing (2009).

27 Bittner (2001, pp. 47-62). Pour un aperçu du débat entre Jean-Paul Brodeur et Egon Bittner, Collectif (2001).

28 Brodeur (2008, p. 264).

29 Nord (2000).

30 L'exemple de l'identification dactyloscopique, d'abord utilisée au Bengale sous domination britannique, est bien connu: Cole (2001); Singha (2000).
} 
cela, revenir sur les modalités des «greffes» d'institutions policières dont elles ont fait l'objet.

\section{LA GREFFE POLICIÈRE ET LES DISPOSITIFS VERNACULAIRES}

Les notions de «transfert» ou de «diffusion de modèles » policiers glissant des métropoles vers les colonies doivent en effet être abandonnées. Comme nous l'avons suggéré, la professionnalisation de la police est volontiers décrite dans les sources produites par les idéologues de l'Empire comme le progrès de l'émancipation bureaucratique des institutions policières européennes contre les résistances culturelles locales et le remplacement progressif du soldat aux colonies par le policier. Dans la bibliothèque coloniale, les succès policiers sont portés au crédit de l'efficacité de l'entreprise coloniale européenne, tandis que bavures et excès de brutalité sont systématiquement imputés aux passions intempestives des cultures indigènes. Le maintien de l'ordre colonial, en effet, est volontiers présenté comme une double rupture: rupture de l'ordre colonial contre un supposé désordre précolonial, d'une part; rupture de l'institution européenne rationnelle et moderne contre des institutions traditionnelles perçues comme archaïques, d'autre part ${ }^{31}$.

L'historiographie récente a pourtant montré que les polices coloniales résultent en réalité d'une toute autre logique: celle de la greffe d'institutions importées sur des institutions vernaculaires qui leur préexistent. Refuser de voir comment les polices coloniales s'articulent aux institutions précoloniales, c'est s'interdire de comprendre l'historicité des dispositifs coloniaux, et, partant, s'enfermer dans l'illusion coloniale de la tabula rasa. Celle-ci connut certes des applications pratiques, le plus souvent éphémères, par exemple dans la ville d'Alger, conquise en 1830, où les dirigeants français décidèrent de rompre totalement avec les institutions ottomanes. Au bout de quelques années, le désordre qui régnait dans une ville autrefois connue pour sa sécurité les obligea à repenser le maintien de l'ordre dans un espace où les corporations de métier et les hiérarchies sociales avaient été bouleversées ${ }^{32}$.

Penser la police comme le résultat de processus de concaténation qui peuvent prendre la forme de «combinatoires impériales $»^{33}$, c'est prendre en compte non seulement les continuités institutionnelles entre les polices précoloniales et les polices coloniales, mais aussi le branchement de celles-ci sur d'autres institutions locales, ainsi que l'invention du policing précolonial par l'institution coloniale. Différentes formes d'articulation des polices coloniales aux dispositifs vernaculaires peuvent ainsi être exposées.

Première d'entre elles: les polices des chefferies. Conformément au principe du gouvernement indirect, les polices de chefferie (Native Authority Police Forces) furent une institution centrale pour le maintien de l'ordre quotidien dans l'empire britannique. En Afrique, à Aden, aux îles Fidji et en Inde, elles cohabitèrent avec d'autres formes de polices plus centralisées et militarisées (Central Government Police Forces par exemple) ${ }^{34}$. Le gouvernement indirect (indirect rule), bien que

\footnotetext{
31 Mbembe (2000, pp. 221-230); Mudimbe (1988, pp. 44-97).

32 Belhamissi (2005); Boyer (1963); Hoexter (1982); Shuval (1999).

33 Bayart, Bertrand, Gordadze, Hibou, Mengin (2006, p. 16).
} 
déjà pratiqué dans les faits dans différents territoires, fut théorisé au lendemain de la Première Guerre mondiale par l'administrateur britannique Frederick Lugard à partir de son expérience de gouverneur au Nigeria. Sur ce territoire, l'Empire de Sokoto, au nord, et les Royaumes yoruba, à l'ouest, avaient laissé des structures bureaucratiques solides sur lesquelles Lugard s'appuya pour établir à moindres coûts la domination coloniale britannique. Dans les dix premières années du vingtième siècle furent ainsi progressivement reconnues par les Britanniques les forces de police locales sous l'autorité des émirs ou des chefs.

Dans les émirats Haussa du nord Nigeria par exemple, l'institution précoloniale des dogarai, ces esclaves gardes-du-corps et messagers de l'Émir, fut reconnue officiellement en 1907 par l'administration britannique ${ }^{35}$. Ce système de "police indigène» fut ensuite étendu au reste du territoire. Bien entendu, l'institution ainsi recréée n'était en rien la simple continuation des polices précoloniales. Les fonctions des dogarai s'accrurent et ils cessèrent d'être les simples dépendants du chef pour devenir des agents permanents rémunérés par l'administration. Ils furent en outre de plus en plus recrutés parmi les grandes familles nobles des émirats, chose impensable avant la période coloniale pour un métier autrefois réservé aux esclaves. Cependant, les dogarai symbolisaient le pouvoir des émirs face au pouvoir central, et ils devinrent une garantie importante du soutien de ces chefs au pouvoir britannique. Il faut ainsi paradoxalement attendre l'indépendance et le coup d'État militaire de 1966 pour voir cette institution dissoute par le pouvoir de Lagos, qui tenta ainsi de centraliser les outils de la violence d'État.

L'influence de la théorie du gouvernement indirect s'étendit à d'autres territoires de l'empire britannique, où des Native Authorities Police Forces furent adoptées dans l'entre-deux-guerres ${ }^{36}$. L'administration coloniale allemande fit également usage des principes de gouvernement indirect et instaura des polices de chef au Togo (Häuptlingspolizisten) dès avant la Première Guerre mondiale. Même dans les colonies françaises, où le gouvernement direct était en principe la règle - à l'exception des protectorats -, l'administration ne put complètement se passer des institutions précoloniales, comme le montre dans ce dossier Nicolas Courtin avec l'exemple de Madagascar. Des expérimentations diverses de gardes indigènes fondées sur la base du village jouèrent un rôle clé dans la pacification du territoire et l'imposition d'un ordre local, lointaine émanation de la domination impériale. Au début du $\mathrm{XX}^{\mathrm{e}}$ siècle, ces polices communautaires étaient souvent non-professionnelles, à l'instar des veilleurs de nuit du Tonkin sous domination française. Comme dans les décennies précédentes, ils étaient chargés de surveiller les récoltes et rendaient compte à un chef de village devant relayer les dispositifs de surveillance administrative et politique mis en place par une puissance coloniale dont l'autorité était soumise à des remises en cause périodiques ${ }^{37}$.

34 Killingray et al. (1991).

35 Rotimi (2001, pp. 1-22).

36 La diffusion des polices de chef à des territoires où les chefs n'avaient, à l'époque précoloniale, pas de corps de police spécialisé - à la différence des États bureaucratisés du nord Nigeria -, montre bien que ces institutions n'avaient parfois d'indigènes que le nom.

37 Voir la thèse en cours de Johann Grémont. Sur le cas du Cambodge, Guérin (2008). 
Deuxième forme de la greffe policière coloniale: l'articulation aux structures sociales, économiques et techniques locales. Cette articulation varie naturellement largement selon les périodes et les territoires, mais, dans l'ensemble, le constat est valable: l'administration coloniale ne peut faire autrement que de reprendre à son compte un certain nombre de structures socio-économiques dont elle prétend pourtant s'affranchir. C'est en particulier le cas des circuits esclavagistes. Dans le dernier quart du XIX ${ }^{\mathrm{e}}$ siècle, de nombreuses forces de police recrutèrent leurs membres en les «libérant» de leurs maîtres, c'est-à-dire en contraignant les marchands d'esclaves à procurer des hommes à la troupe de police, et en forçant ces nouvelles recrues à rembourser leur prix d'achat par prélèvement d'une somme régulière sur leur salaire ${ }^{38}$. Les anciens marchés à esclaves devinrent des lieux incontournables du recrutement policier. La ville de Salaga en Afrique de l'Ouest en fournit un bon exemple $^{39}$. À la fin du XIX ${ }^{\mathrm{e}}$ siècle, cette ville fut décrite comme le «plus grand marché d'esclaves du Soudan occidental $»^{40}$, d'où partaient annuellement 15000 esclaves échangés contre de la cola, du sel ou des produits manufacturés européens $^{41}$. La Gold Coast Constabulary britannique et la Polizeitruppe allemande venaient toutes deux s'y approvisionner en jeunes recrues ${ }^{42}$. Dans ce type de processus, les intermédiaires locaux - et en particulier les marchands d'esclaves, comme le fameux Tippo Tipp en Afrique orientale -, sont incontournables ${ }^{43}$.

Même après la fin des recrutements systématiques d'esclaves, au début du $\mathrm{XX}^{\mathrm{e}}$ siècle, les réseaux locaux continuèrent d'être une ressource fondamentale pour les polices coloniales. Les routes et pistes empruntées par les caravanes de marchands furent les premiers axes utilisés par les policiers pour contrôler les territoires. Certes, les nouveaux axes coloniaux, notamment ceux tracés par le chemin de fer, découpèrent des espaces dictés par des ambitions économiques mais également par des besoins stratégiques ${ }^{44}$. Toutefois, l'État colonial resta longtemps cantonné à des «îles de domination» autour des stations, et adossé aux réseaux locaux ${ }^{45}$. Il faudrait faire l'inventaire des techniques autochtones - usage extensif du portage, expéditions en caravanes, usages du chameau - adoptées par les polices coloniales pour rendre véritablement compte des diverses facettes de cette greffe ${ }^{46}$. On se contentera

38 L'exemple des esclaves de la troupe de police du Cameroun allemand a été analysé dans: Oloukpona-Yinnon (1987).

39 Johnson (1986).

40 Cité par Johnson (1986, p. 341).

${ }^{41}$ Une estimation de Curt von François, qui, comme les autres observateurs de l'époque estime le nombre d'esclaves à partir du nombre de caravanes qu'il avait vu arriver en ville, Johnson (1986, p. 345).

42 Krause, Gustav Adolf, Hausa-Soldaten in der englischen Goldküsten-Kolonie, in: Neue preußische Zeitung, Nr. 20, 12. janvier 1889, p.2.; Krause, Gustav Adolf, Was bringen die Händler aus Salaga nach der Togo-Küste? Eine Frage an die Regierung, in: Neue preußische Zeitung, Nr.247, 27 mai

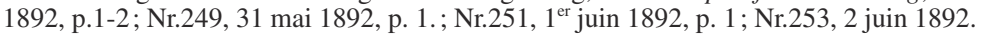

43 Pesek (2006, p. 304).

44 Killingray (1999b).

45 L'expression d' «îles de domination» fut proposée par Michael Pesek pour décrire le pouvoir géographiquement limité de l'État colonial à la fin du XIX ${ }^{\mathrm{e}}$ siècle et au début du XX $\mathrm{XX}^{\mathrm{e}}$ siècle. Cf. Pesek (2006, p. 244).

46 Frémeaux (1995). 
pour le moment de rappeler, avec le sociologue Trutz von Trotha, qu'une pratique comme la «tournée», clef de voûte incontournable de l'exercice du pouvoir des «rois de la brousse» qu'étaient les commandants de cercle accompagnés de leurs gardes-cercles, ressemble étrangement à la tournée des chefs africains de l'époque précoloniale ${ }^{47}$.

Troisième élément de l'articulation policière coloniale sur les mondes précoloniaux : la question de l' «invention de la tradition». L'argument, proposé en 1983 par Eric Hobsbawm et Terence Ranger, est connu : les empires ont assis leur domination sur un ensemble de pratiques et de représentations qui, se donnant comme anciennes et par là même dignes de déférence, étaient en réalité largement et consciemment construites par les bureaucraties impériales ${ }^{48}$. On pense bien sûr à l'ensemble des représentations des agents de l'ordre, et en particulier aux uniformes (la chéchia des tirailleurs, le fez des gardes-cercles, le chapeau pointu de la police allemande de Kiautschou, le pagne de la police allemande de Nouvelle Guinée, le turban des troupes britanniques de l'Inde) ou aux insignes des Corps (étoile et croissant des gardes-cercles; croissant, devise arabe et main de Fatima du premier régiment de tirailleurs algériens) qui suggèrent à moindre coût une continuité avec les systèmes symboliques vernaculaires. On pense, aussi, à ces corps policiers légitimés par un label à coloration locale, des régiments de Spahis algériens aux Goumiers marocains, en passant par les Ruga Ruga ou les Askaris d'Afrique orientale allemande. De ce point de vue, la mythologie associée à la figure du Maréchal Lyautey au Maroc emprunte largement à ce répertoire symbolique qui minimise les bouleversements imposés par l'action des forces de l'ordre colonial. Cela ne fait pas des polices coloniales les héritières des dispositifs vernaculaires, mais diffuse à travers l'Empire le mythe de forces d'un ordre certes nouveau mais respectueux des traditions et visant un certain degré de consentement des populations colonisées.

L'exemple le plus probant - et le mieux étudié -, et qui suffira ici à rendre compte de la complexité d'appareils policiers coloniaux qui ne sont ni les prisonniers des traditions locales, ni un avatar exotique des polices métropolitaines, est celui des «races guerrières ». L'idée de «race guerrière » c'est-à-dire la croyance selon laquelle certains groupes sont physiologiquement ou culturellement prédisposés à servir dans les forces armées ou dans les forces de l'ordre, est, sous sa forme moderne, née dans l'Empire britannique durant la seconde moitié du XIX ${ }^{\mathrm{e}}$ siècle $^{49}$. Au début de ce siècle, les officiers britanniques recrutaient leurs soldats et officiers indigènes parmi l'élite Hindou des hautes «castes» du nord de l'Inde. En 1857, une révolte de ces soldats et officiers conduisit les administrateurs britanniques à repenser leur politique de recrutement, et à exclure progressivement les élites hindoues des institutions militaires. Ils leurs préférèrent les Sikh du Penjab, qui ne formaient pas une ethnie distincte mais plutôt un groupe uni par des pratiques religieuses, en particulier celles de la confrérie spirituelle et militaire Khalsa. La littérature coloniale fit des Sikh une race reconnaissable par des caractéristiques physiques et morales distinctes (parmi lesquelles la franchise, l'obstination et le courage physique), et justifia ainsi d'avoir recours principalement à eux. L'idée de «race mar-

\footnotetext{
47 Trotha (1994, pp. 117-143).

48 Hobsbawm (1983); Ranger (1983, 1993).

49 Streets (2004).
} 
tiale» fit son chemin, aux Indes d'abord. Les «Gurkha» du Népal furent, encore plus clairement que les Sikh, un groupe ethnique créé de toutes pièces, à partir de recrues issues de divers ensembles culturels, au sein de l'armée britannique ${ }^{50}$. Dans le reste de l'empire britannique, ensuite: Highlanders en Écosse, Kamba en Afrique orientale britannique, Haussa et Konkomba en Gold Coast. Dans les empires concurrents et envieux du succès britannique, enfin: Soudanais en Afrique orientale allemande, Zulu au Mozambique portugais, Bambara en Afrique occidentale française $^{51}$.

Certes, les «martial races» furent d'abord une invention militaire. La propension à combattre est un critère déterminant des définitions coloniales des ethnies guerrières. Mais en raison des circulations importantes entre armées et polices coloniales - circulations d'hommes, d'idées et confusion des fonctions -, l'étude des polices ne peut faire l'économie du concept. La notion de «race guerrière» n'est pas, en effet, la simple traduction militaire des théories raciales d'une époque: c'est une manière consciente d'utiliser le langage de la race pour défendre des objectifs politiques et pratiques différents selon les endroits, et d'inventer ainsi, a posteriori, des traditions autochtones pour les besoins du maintien de l'ordre colonial. David Arnold a montré combien les priorités de recrutement des polices dans le district de Madras, en termes de castes (les intouchables étant d'abord surreprésentés puis, à partir des années 1860, délaissés car inaptes à obtenir le consentement minimal à la police du quotidien) et de religions (les musulmans représentant en moyenne plus d'un quart des policiers recrutés) pouvaient varier selon les lieux et les époques.

Cette politique des populations contribua donc aux césures du corps social, qu'elle renforça cependant plus qu'elle ne créa: ainsi, parmi les groupes paysans fortement présents dans la police de Madras, nombreux étaient ceux qui se référaient, bien avant la colonisation, à un glorieux passé militaire. Ce branchement des institutions policières «anglaises» sur des groupes, des représentations, voire des institutions (les chefs de village et leurs assesseurs) ne doit pas être surestimé: dès le milieu du XIX ${ }^{\mathrm{e}}$ siècle, il apparut ainsi que la nouvelle gouvernementalité, notamment liée à l'industrialisation de certaines villes indiennes, nécessitait «l'établissement d'une nouvelle police $»^{52}$. C'était d'autant plus le cas dans les colonies de peuplement où les nouveaux arrivants d'Europe étaient les cibles premières de l'action des polices urbaines qui, parfois, ne faisaient pas même mention de la présence d'indigènes dans des villes aux espaces ségrégés. Dans les campagnes, les colons avaient de véritables pouvoirs de police et un droit quasi-illimité à l'usage de la force sur des colonisés qui avaient par ailleurs bien plus affaire aux militaires qu'à des forces de police concentrées dans les quartiers européens des grandes villes ${ }^{53}$.

\footnotetext{
50 «As a 'race', the Gurkhas can truly be said to have been both produced and maintained in the Indian army and nowhere else». Streets (2004, p. 9).

51 Parsons (1999); Killingray (1999b); Lunn (1999); Morlang (2008, p. 16).

52 Arnold (1986, p. 13).

53 Anderson (1994).
} 


\section{DE LA PACIFICATION ET DU CONSENTEMENT EN SITUATION COLONIALE}

Dès que l'on rompt avec les considérations en termes de «modèles » pour se pencher sur la fabrique de l'ordre colonial, il devient difficile de recourir à un récit linéaire décrivant un progressif passage depuis un ordre assuré par les militaires vers un ordre plus civil et pacifié assuré par des policiers. Le détour colonial permet ainsi de reposer les questions de la monopolisation et des limites de l'usage de la force. Il n'est d'ailleurs pas anodin que le débat contemporain, en particulier les échanges entre Egon Bittner et Jean-Paul Brodeur sur l'opposition entre contrainte et consentement, soit notamment nourri par l'attention portée aux populations autochtones du Canada et à d'autres situations (Irak, Afghanistan...) que l'on pourrait qualifier de (post)-coloniales ${ }^{54}$. La dichotomie/complémentarité entre polices et armées, qui est au cœur de l'approche classique de la professionnalisation policière, doit alors être relativisée par un ensemble d'observations: certains corps policiers, telle la gendarmerie, ont un statut militaire et peuvent être engagés sur des théâtres d'opération extérieurs, dans un contexte de guerre; les militaires ont longtemps participé - en France, encore, pendant les grèves de 1947-1948 (Mencherini, 1998) - au maintien de l'ordre intérieur sans forcément faire usage d'une force qui serait supérieure ou plus létale que celle des polices qu'ils suppléent ou soutiennent ${ }^{55}$; l'indistinction entre opérations de police et situation de guerre est consubstantielle à certains conflits marqués «par des situations anormales entre la guerre et la paix, où les deux termes sont confondus $»^{56}$, en particulier en situation coloniale ${ }^{57}$.

Ces réflexions invitent à se poser la question de la possibilité même d'une police en situation coloniale. En effet la «pacification» de nombreux territoires ne fut jamais achevée et la plupart des historiens actuels des colonisations s'attachent à remettre en cause la distinction longtemps canonique entre le moment de la conquête et celui de la construction de l'État colonial, symbolisée par la transition des pouvoirs militaires aux autorités civiles ${ }^{58}$. De plus, l'idée selon laquelle «la police ne peut opérer que sur la base d'un consentement de la population dans sa totalité et de ses parties distinctes à se soumettre à son autorité ${ }^{59}$ pose manifestement problème en situation coloniale ${ }^{60}$. La notion de policing by consent fut en effet forgée dans le contexte anglais d'une police non armée - en tout cas dépourvue au quotidien d'armes à feu, depuis 1829 dans le cas londonien - où la figure mythifiée du bobby apparaissait comme un simple «citoyen en uniforme», armé de sa seule autorité consentie, de son «tact et de son sens de l'humour ${ }^{61}$.

\footnotetext{
54 Bittner (2001); Brodeur (2001).

55 Johansen (2001).

56 Schmitt (1992, pp. 163-167).

57 Si, dans sa Théorie du partisan, Carl Schmitt utilise largement les exemples des guerres d'Indochine et d'Algérie, ses réflexions trouvent leur origine dans la «guérilla menée par le peuple espagnol de 1808 à 1813 » contre les troupes d'occupation de Napoléon. Schmitt (1992).

58 «La domination coloniale fut plus que toute autre chose une entreprise de conquête ininterrompue», Bernault (1999, p. 62).

59 Brodeur (2008, p. 264).

60 Anderson, Killingray (1991, p. 9).

61 Waddington, Wright (2009, p. 468).
} 
Même si un certain nombre de polices coloniales de l'empire britannique suivirent le modèle londonien et ne furent pas dotées d'armes à feu - ainsi les constables de Madras au XIX ${ }^{\mathrm{e}}$ siècle $^{62}-$, l'absence d'une configuration liant ordre policier apparemment démilitarisé et ordre politique fondé sur une citoyenneté partagée ${ }^{63}$ oblige à s'interroger sur la pertinence de la notion de «consentement à l'autorité policière» en contexte colonial. Depuis trois décennies, les chercheurs qui se sont penchés sur la question apportent d'ailleurs une réponse quasi unanime: il s'agit de rompre avec des perspectives qui jusqu'alors avaient insisté sur l'émergence de forces de police symboles d'une juridicisation du gouvernement colonial et garantes de l'obligation de respect de la loi ${ }^{64}$. Dans un recueil centré sur la problématique de la «fabrication du consentement en contexte colonial ${ }^{65}$, David Anderson confirme les conclusions du groupe initial des subaltern studies, pour qui le gouvernement colonial s'était caractérisé par une «domination sans hégémonie ${ }^{66}$. Selon lui, le concept d'hégémonie ne s'applique pas aux forces de police en situation coloniale: autrement dit, l'intériorisation de la «violence douce» et autres mécanismes de domination symbolique ne prirent jamais le pas sur une «violence directe $»^{67}$ qui resta au cœur des pratiques policières ${ }^{68}$. Depuis la fin des années 1990, certains historiens indiens ont cependant pris leur distance avec l'accent mis dans les subaltern studies sur la violence comme mode de domination coloniale et ont insisté sur une police du quotidien fondée sur des arrangements et des transactions collusives avec les habitants, même issus des quartiers les plus pauvres ${ }^{69}$. Il semble cependant que pour cette aire coloniale, incontestablement la plus analysée sur le plan du colonial policing, un consensus se dégage: si la faiblesse des moyens policiers était réelle, leurs connexions avec les forces armées étaient primordiales. Surtout, dans les interactions quotidiennes, les capacités d'action des colonisés ne doivent pas faire oublier l'asymétrie des relations de pouvoir ${ }^{70}$ et la permanence d'un mode de gouvernement qui «impliquait nécessairement le recours à la violence» dans la plupart des régions colonisées ${ }^{71}$.

Si le mouvement de spécialisation et de «professionnalisation» policière s'observe à des dates et selon une chronologie diverses suivant les régions ${ }^{72}$, il reste que

${ }^{62}$ À l'exception d'une section par district, Arnold (1986, p. 25).

63 En Grande-Bretagne même, ce n'est cependant qu'en 1918 que l'ensemble des ouvriers fut intégré au corps électoral (à la même date, les femmes de plus de 30 ans obtinrent le droit de vote).

64 Arnold (1986, p. 3). Quelques auteurs continuent cependant d'insister sur le «law enforcement» et l'implantation progressive d'un ordre juridique libéral. Voir par exemple Wiener (2008).

65 Engels, Marks (1994, p. 14).

66 Guha $(1982,1997)$.

67 Les notions de «violence directe» et de «violence douce» (synonyme de violence symbolique chez Pierre Bourdieu) sont utilisées en français dans le texte en anglais. Engels, Marks (1994, p. 1).

68 Anderson (1994, p. 264). David Anderson ne rejette pas le concept d'hégémonie, inspiré de Gramsci, quand il s'applique à d'autres dispositifs de pouvoir (l'éducation, la médecine...) que la police.

69 Chandavarkar (1998).

70 Kidambi (2007).

71 Thioub (1999, p. 287).

72 Les articles du dossier montrent bien la tentation constante de dupliquer aux colonies une partie des organigrammes métropolitains et d'y implanter certains services spécialisés (police des mœurs, identification judiciaire...). 
les colonies, quel que soit leur statut, sont marquées par la visibilité du soutien que l'armée apporte aux maigres forces de police. De plus, l'importance des unités paramilitaires, dont le rôle s'accroît au fur et à mesure de l'institutionnalisation d'unités policières différenciées, est aussi notable ${ }^{73}$. Hormis dans les grandes villes, l'immense majorité des habitants reste cependant peu confrontée à des forces armées qui, quand elles interviennent, le font dans une indistinction et un amalgame entre militaires et policiers: au Dahomey, des «tournées de police» étaient ainsi organisées suite à un incident sur un territoire rural et visaient à «ramener le calme» en déployant à la fois des forces de police, les gardes de cercle, et des éléments militaires, les compagnies de tirailleurs. Quand l'incident virait au conflit ouvert et généralisé, ces opérations de police se transformaient en une véritable expédition miliaire $^{74}$. Les forces de police sont donc bien au cœur d'une «pacification» jamais achevée: même les analystes les plus empressés à voir dans les policiers aux colonies les défenseurs du «rule of law» reconnaissaient que ces derniers devaient cependant pouvoir user d'une force illimitée afin de prendre en compte les «circonstances spécifiques » de la situation coloniale ${ }^{75}$.

Cette force, qui peut difficilement être qualifiée de légitime tant elle est exogène aux sociétés colonisées - quand bien même elle est mise en œuvre par des indigènes travaillant pour la puissance coloniale -, n'est pas non plus monopolisée par les unités policières, ni même militaires. Le mouvement de monopolisation de l'usage d'une force que nul individu ou organisation ne peut excéder, caractéristique du mandat policier, n'est pas vérifié dans les empires coloniaux. Au fur et à mesure des décennies, les autorités cherchèrent certes à réguler et à poursuivre les usages privés (par des agents de l'État ou des particuliers) des violences extrêmes ${ }^{76}$ mais, dans les colonies de peuplement, tout au long des $\mathrm{XIX}^{\mathrm{e}}$ et $\mathrm{XX}^{\mathrm{e}}$ siècles, les «Européens » étaient dans les faits tous dotés de pouvoirs de police, en particulier celui de se «faire justice» eux-mêmes à l'encontre de leurs personnels indigènes ${ }^{77}$. Quand ils se sentaient menacés, ils pouvaient même créer des milices qui, fondues au sein des forces de l'ordre, avaient pouvoir de vie ou de mort sur les colonisés: c'est ainsi qu'en mai 1945, dans la région de Guelma (Algérie), la milice locale, dirigée par le sous-préfet et ancien commissaire de police, fut directement responsable de centaines de morts et de disparus pendant les «événements du Constantinois » ${ }^{78}$.

Cette perméabilité des forces de police aux milices en armes et aux unités militaires ne doit pas faire oublier que les policiers eux-mêmes usaient d'une violence extrême qui ne débordait pas mais, au contraire, définissait le cadre de certaines de leurs actions. C'est ainsi que les techniques de maintien de l'ordre ne connurent pas exactement les même évolutions en métropole qu'aux colonies: si la question de la pacification du maintien de l'ordre ne fait guère de doute en ce qui concerne les réponses apportées aux démonstrations du mouvement ouvrier français ou britannique $^{79}$, « une mitraillade dans la foule colonisée faisait hausser les épaules [du colo-

Arnold (1986, p. 234).

Voir la thèse en cours de Bénédicte Brunet-Laruche.

Curry (1932, pp. 9, 86).

Bailkin (2006); Wiener (2008).

Anderson (1994, p. 252).

Peyroulou (2009).

Bruneteaux (1996); Waddington (1998). 
nisateur $] \gg^{80}$ et nombre de dirigeants adhéraient à l'idée du commissaire en chef de Bombay, en 1929, selon laquelle «le seul remède à l'émeute est de tirer ${ }^{81}$. Cette propension à faire usage des armes ne peut pas être reliée à une supposée faiblesse d'un État colonial, et de ses forces de maintien de l'ordre, contraints de répondre à la contestation par une violence disproportionnée: elle s'observe aussi, dans le cas français, vis-à-vis des manifestants colonisés émigrés en métropole. C'est ainsi qu'à Paris les seuls manifestants tués par balles policières après la Seconde Guerre mondiale étaient des Algériens, en particulier au cours de la manifestation du 14 juillet $1953^{82}$. Le mouvement de civilisation des mœurs policières, souvent pensé en lien avec la professionnalisation des techniques de maintien de l'ordre, était donc « compartimenté ${ }^{83}:$ il ne touchait pas certaines populations victimes de stéréotypes négatifs et d'un mode de domination en partie fondé sur l'hypothèse de l'inégalité des races. En juillet 1953, les innovations organisationnelles apportées à ce qui était perçu comme la réponse nécessaire à «l'émeute» fomentée par les colonisés émigrés en témoignent: certaines unités furent spécialisées dans la répression de la «criminalité nord-africaine ${ }^{84}$, d'autres professionnalisées dans l'exercice du maintien de l'ordre. Ces compagnies de district furent en pointe dans les répressions meurtrières du 17 octobre 1961 et du 8 février $1962^{85}$ : à tout le moins, la professionnalisation, certes inachevée à l'aune des standards des CRS ou des gendarmes mobiles, des techniques de maintien de l'ordre n'empêchait pas que la violence se déchaîne contre les colonisés et leurs soutiens. Cette spécialisation d'effectifs dans la répression de populations stigmatisées et ciblées comme ennemis politiques était porteuse d'une élévation des seuils de violence mobilisables.

En matière de pratiques répressives qui, en certaines circonstances, pouvaient s'apparenter à de véritables «pogroms $»^{86}$, on observe ainsi un véritable répertoire d'action policier, qui, de décembre 1952 à Casablanca à octobre 1961 à Paris, unit le centre et la périphérie de l'empire ${ }^{87}$. Les circulations d'acteurs, de savoirs ou d'innovations organisationnelles au sein de l'empire ne sont certes pas sans expliquer ces correspondances dans les dispositifs répressifs ${ }^{88}$, mais il ne faudrait pas en tirer la conclusion que seuls les agents ayant une expérience impériale étaient directement impliqués dans les tueries de manifestants colonisés. Ainsi, le 14 juillet 1953,

80 Memmi (1985, p. 105).

81 «The only medicine for the rioters was the bullet» affirma-t-il après les émeutes inter-communautaires de 1929. Cité in Chandavarkar (1998, p. 213).

82 En fait, un des sept tués était un militant syndical (CGT) parisien s'étant mêlé au cortège des nationalistes algériens assailli par la police. Un autre manifestant algérien avait déjà été tué par balles au cours de la manifestation parisienne du 28 mai 1952 contre la visite du général Ridgway (général en chef de l'OTAN).

83 Le concept de «compartimentation» est emprunté à Abram de Swaan, qui l'utilise afin de complexifier les interprétations de la théorie eliassienne de la «civilisation des mœurs» et d'y intégrer la question de «l'extermination de masse» au XX $X^{\mathrm{e}}$ siècle. De Swaan (2003).

84 Blanchard (2006, 2008, pp. 500-513).

85 Dewerpe (2006, pp. 170-192).

86 Le terme «pogrom» a notamment été utilisé par l'historien Pierre Vidal-Naquet pour qualifier le massacre du 17 octobre 1961: Vidal-Naquet (1987). Sur la répression policière en octobre 1961: House, MacMaster (2006).

87 Blanchard (2008, pp. 660-663); House (2011).

88 Sinclair, Williams (2007). 
ceux qui tirèrent sur les nationalistes algériens du MTLD étaient des gardiens de la paix de la préfecture de police ${ }^{89}$. Le maintien de l'ordre de la «manifestation traditionnelle» du 14 juillet avait d'ailleurs été organisé selon les modalités et avec les unités habituelles. Ce n'était donc pas seulement outre-mer que le mouvement de professionnalisation policière s'accommodait de formes de brutalisation des populations colonisées et d'une pacification aussi inachevée que compartimentée.

\section{UNE BRUTALISATION DES SOCIÉTÉS VERNACULAIRES?}

Le détour par l'empire et les situations coloniales permet ainsi de complexifier l'analyse des professionnalités policières et de ne pas les appréhender dans une perspective évolutionniste ou selon le schéma simplifié du processus de civilisation. Il s'avère que les différents processus généralement réunis dans le mouvement de professionnalisation peuvent s'articuler en des configurations variant selon les contextes, sans détenir en eux-mêmes des effets prédéterminés, qu'ils soient pacificateurs ou brutalisateurs. Par ailleurs, les espaces impériaux et métropolitains sont si connectés qu'ils apparaissent chacun marqués de stases, brèches et compartimentations croisées qui interdisent de tirer à leur propos des conclusions trop homogènes - sans qu'il faille négliger pour autant les dynamiques propres qui les animent. Une cartographie, subtile, reste à faire, mais dans cette perspective, l'analyse des phénomènes paraît moins donnée à l'avance et semble devoir se soumettre à de constants ajustements.

Un même dispositif peut ainsi être perçu de manière différente en métropole ou aux colonies: alors qu'en France métropolitaine la pratique consistant à éloigner les commissaires de police de leur communauté locale a été analysée comme un signe de la professionnalisation policière (toujours comprise comme souhaitable et positive) au cours de la première moitié du $\mathrm{XIX}^{\mathrm{e}}$ siècle $^{90}$, le fait qu'aux colonies les recrutements se soient souvent faits sur une base ethnique et géographique, visant à la distance avec les populations locales, a été un des facteurs des relations tendues avec les populations. Il s'agissait cependant là aussi d'une caractéristique d'une professionnalisation policière en rupture avec les formes de maintien de l'ordre communautaires vernaculaires. Ces méthodes continuent d'ailleurs de marquer certains contextes post-coloniaux dans lesquels les forces de l'ordre sont sciemment recrutées dans des groupes linguistiques différents de ceux des populations dont elles sont en charge $\mathrm{e}^{91}$. Dans ces configurations, des pratiques considérées comme dysfonctionnelles par rapport aux normes occidentales de professionnalité (absence d'enquête, participation des «victimes » aux arrestations...) sont en fait à la fois des legs du passé colonial et «une nécessaire localisation et adaptation des routines policières $»^{92}$. Il apparaît dès lors que les voies de la professionnalisation empruntées pendant la période coloniale ont laissé une empreinte durable et permettent de relativiser les affirmations sur les nouvelles criminalités et réponses sécuritaires dans

\footnotetext{
89 Au moins huit gardiens de la paix reconnurent avoir fait usage de leur arme de service, plusieurs centaines furent impliquées dans les affrontements avec les manifestants.

90 Merriman (2006, p. 7).

91 Voir l'exemple du Nord-Ghana étudié par Jan Beek (2010, p. 284).

92 Beek (2010, p. 280).
} 
certaines villes du Sud, en particulier africaines (Fourchard, Albert 2003; Fourchard 2008). Ces trajectoires historiques de la professionnalisation policière sont d'ailleurs souvent dénoncées comme ayant acclimaté les sociétés post-coloniales à des usages de la violence et de la prévarication qui sont, aujourd'hui comme hier, partie prenante d'un mode global de gouvernement ${ }^{93}$.

Emmanuel Blanchard CESDIP

Immeuble Edison 43, boulevard Vauban F - 78280 Guyancourt blanchard@ cesdip.fr

Quentin Deluermoz Université Paris 13 99 av J.B. Clément, F- 93430 Villetaneuse quentin.deluermoz@gmail.com

Joël Glasman Humboldt-Universität zu Berlin Institut für Asien- und Afrikawissenschaften Seminar für Afrikawissenschaften

Unter den Linden 6 D-10099 Berlin glasmanj@cms.hu-berlin.de

\section{BIBLIOGRAPHIE}

Abbott, A., The System of Professions. An Essay on the Division of Expert Labour, Chicago and London, University of Chicago Press, 1988.

Ahire, P., Imperial Policing: The Emergence And Role Of The Police In Colonial Nigeria 1860-1960, Milton Keynes, Open University Press, 1991.

Anderson, D., Policing the Settler State: Colonial Hegemony in Kenya, 1900-1952 in Engels, D., Marks, S., Contesting Colonial Hegemony. State and Society in Africa and India, London, British Academic Press/The German Historical Institute London, 1994, pp. 248-264.

Anderson, D., Killingray, D., Consent, coercition and colonial control: policing the empire, 1830-1940», in Anderson, D., Killingray, D., Policing the empire. Government, authority and control, 1830-1940, Manchester, Manchester University Press, 1991, pp. 1-16.

Arnold, D., Police Power and Colonial Rule, Madras, 1859-1947, Oxford, OUP, 1986.

Bailkin, J., The Boot and the Spleen: When Was Murder Possible in British India?, Comparative Studies in Society and History, 2006, 48, 2, pp. 462-493.

Bayart, J.-F., Bertrand, R., Gordadze, T., Hibou, B., Mengin, F., Legs colonial et gouvernance contemporaine, Document de Travail de l'Agence Française de Développement, 2006, $13,1-22$.

93 Arnold (1986); Dhillon (2005); Verma (1999). 
Beek J., Étiqueter les «déviants»: le travail des policiers au Nord-Ghana, Déviance et Société, 2010, 34, 2, pp. 279-290.

Belhamissi, M., La police d'Alger au début de l'ère coloniale, El Watan, 2005, [http://www. elwatan.com/La-police-d-Alger-au-debut-de-1].

Berlière, J.-M., La professionnalisation: revendication des policiers et objectifs des pouvoirs au début de la III $^{\text {e }}$ République, Revue d'Histoire Moderne et Contemporaine, 1990, 3, pp.398-428.

Berlière, J.-M., Le monde des polices en France, Bruxelles, Complexe, 1996.

Berlière, J.-M., Denys, C., Kalifa, D., Milliot, V. (dir.), Métiers de police. Être policier en Europe, XVIII $-X X^{e}$ siècles, Rennes, PUR, 2008.

Bernault, F., De l'Afrique ouverte à l'Afrique fermée: comprendre l'histoire des réclusions continentales, in Bernault, F., Enfermement, prison et châtiments en Afrique. Du XIX $X^{e}$ siècle à nos jours, Paris, Karthala, 1999, pp. 15-64.

Bittner, E., Florence Nightmale à la poursuite de Willie Sutton. Regard théorique sur la police, Déviance et Société, 2001, 25, 3, pp. 285-306.

Blanchard, E., Police judiciaire et pratiques d'exception pendant la guerre d'Algérie, Vingtième siècle. Revue d'histoire, 2006, 90, pp. 61-72.

Blanchard, E., Encadrer des «citoyens diminués». La police des Algériens en région parisienne, 1944-1962, thèse d'histoire sous la dir. de J.-M. Berlière, Dijon, Université de Dijon, 2008.

Boyer, P., La vie quotidienne à Alger à la veille de l'intervention française, Paris, Hachette, 1963.

Brodeur, J.-P., Le travail d'Egon Bittner: une introduction à la sociologie de la force institutionnalisée, Déviance et Société, 2001, 25, 3, pp. 307-323.

Brodeur, J.-P., Que dire maintenant de la police?, in Ocqueteau, F. (dir.), Notes inédites sur les choses policières, 1999-2006 suivi de Le sociologue, la politique et la police, Paris, La Découverte, 2008, pp. 255-268.

Bruneteaux, P., Maintenir l'ordre, Paris, Presses de la FNSP, 1996.

Champy, F., La sociologie des professions, Paris, PUF, 2009.

Chandavarkar, R., Imperial power and popular politics: class, resistance and the state in India, c. 1850-1950, Cambridge, CUP, 1998.

Cole, S., Suspect Identities. A History of Fingerprintings and Criminal Identification, Cambridge Mass, Havard University Press, 2001.

Collectif, Faire autorité dans la France du XIX ${ }^{\mathrm{e}}$ siècle, Mouvement Social, 2008, 224, numéro spécial.

Collectif, La violence d'État. Les fragiles naissances du maintien de l'ordre en France (18001930), Déviance et Société, 2008, 32, 1, dossier spécial.

Collectif, Norbert Elias et le $\mathrm{XX}^{\mathrm{e}}$ siècle: le processus de civilisation à l'épreuve, Vingtième Siècle. Revue d'Histoire, 2010, 106, numéro spécial.

Curry, J.-C., The Indian police, Faber \& Faber, London, 1932.

De Swaan, A., La dyscivilisation, l'extermination de masse et l'État, in Bonny, Y., Neveu, E., Norbert Élias et la théorie de la civilisation: lectures et critiques, Rennes, PUR, 2003, pp.63-73.

Deluermoz, Q., Les Policiers en tenue dans l'espace parisien (1854-1913): la construction d'un ordre public, thèse d'histoire sous la dir. de D. Kalifa, Paris, Université Paris I, 2006.

Deluermoz, Q., Présences d'État. Police et société à paris (1854-1880), Annales. Histoire, sciences sociales, 2009, 64, 2, pp. 435-460.

Denys, C., Police et sécurité au XVIII siècle dans les villes de la frontière franco-belge, Paris, L'Harmattan, 2002. 
Dewerpe, A., Charonne, 8 février 1962: anthropologie historique d'un massacre d'État, Paris, Gallimard, 2006.

Dhillon, K., Police and politics in India: colonial concepts, democratic compulsions. India police 1947-2002, New Delhi, Manohar, 2005.

Dubar, C., Tripier, P., Sociologie des professions, Paris, A. Colin, 1998.

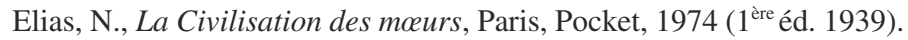

Elias, N., La dynamique de l'Occident, Paris, Pocket, 1975 (1 $1^{\text {ère }}$ éd. 1939).

Emsley, C., Reinke, H., Lévy, R., Les polices aux XIX et $\mathrm{XX}^{\mathrm{e}}$ siècles: aperçus sur les historiographies anglaise, allemande et française, Les cahiers de la sécurité intérieure, $3^{\mathrm{e}}$ trimestre 1994, 17, 13-33.

Engels, D., Marks, S., Hegemony in a colonial context, in Engels, D., Marks, S. (eds), Contesting Colonial Hegemony. State and Society in Africa and India, London, British Academic Press, The German Historical Institute, 1994, pp. 1-15.

Fourchard, L., A new name for an old practice: vigilantes in south-western Nigeria. Africa: The Journal of the International African Institute, 2008, 78, 1, pp. 535-558.

Fourchard, L., Albert, I., Sécurité, crime et ségrégation dans les villes d'Afrique de l'ouest du XIX siècle à nos jours, Paris, Karthala, 2003.

Frémeaux, J., L'Afrique à l'ombre des épées, 1830-1930, Des établissements côtiers aux confins sahariens, Service historique de l'armée de terre, Paris, 1995.

Glasman, J., Penser les intermédiaires coloniaux. Note sur les dossiers de carrière de la police du Togo, History in Africa, 2010, 37, pp. 51-81.

Gooptu, N., The politics of the urban poor in early twentieth-century India, Cambridge, CUP, 2001.

Guérin, M., Paysans de la forêt à l'époque coloniale: la pacification des habitants des Hautes Terres du Cambodge, Rennes-Paris, AHSR_EFEO, 2008.

Guha, R. (ed.), Subaltern Studies I. Writings on South Asian History and Society, New Delhi, Oxford University Press, 1982.

Guha, R., Dominance without hegemony: History and power in colonial India, New York, Harvard University Press, 1997.

Hobsbawm, E., Inventing Tradition, in Hobsbawm, E., Ranger, T. (Eds), The Invention of Tradition, Cambridge, CUP, 1983, pp. 1-14.

Hoexter, M., La Shurta, ou la répression des crimes à Alger à l'époque turque, Studia Islamica, LVII, 1982, pp. 117-146.

House, J., L'impossible contrôle d'une ville coloniale?, Casablanca, décembre 1952, 2011 (à paraître).

House, J., MacMaster, N., Paris 1961 : Algerians, State Terror, And Memory, Oxford, OUP, 2006.

Houte, A.-D., Le Métier de gendarme au XIXe siècle, Rennes, PUR, 2010.

Hughes, E., Le regard sociologique. Essais choisis, Paris, EHESS, 1996.

Jeffries, C., The colonial Police, London, Max Parrish, 1952.

Jobard, F., Bavures policières? La force publique et ses usages, Paris, La Découverte, 2002.

Johansen, A., Violent repression or modern strategies of crowd management soldiers as riot police in France and Germany, 1890-1914, French History, 2001, 15, 4, pp. 400-420.

Johnson, M., The Slaves of Salaga, The Journal of African History, 1986, 27, 2, pp. 341-362.

Karpik, L., Les Avocats: entre l'État, le public et le marché, XIII ${ }^{e}-X X^{e}$, Paris, Gallimard, 1995.

Kidambi, P., The making of an Indian metropolis. Colonial Governance and Public Culture in Bombay, 1890-1920, Aldershot, Ashgate Publishing, 2007. 
Killingray, D., Guardians of Empire, in Killingray, D., Omissi, D., Guardians of Empire, The Armed Forces of The Colonial Powers c.1700-1964, Manchester, MUP, 1999a, pp. 1-24.

Killingray, D., Imagined Martial Communities: Recruiting for the Military and Police in Colonial Ghana, 1860-1960 in Lentz, C., Nugent, P., Ethnicity in Ghana: the Limits of Invention, London, Macmillan, 1999b, pp. 119-136.

Killingray, D., Anderson, D., An orderly retreat? Policing the end of empire, in Killingray, D., Anderson, D., Policing and Decolonisation. Nationalism, Politics and the Police, 19171965, Manchester, MUP, 1991, pp. 1-21.

Knöbl, W., Polizei und Herrschaft im ModernisierungsproseSS. Staatsbildung und innere Sicherheit in PreuSSen, England und Amerika 1700-1914, Frankfurt, Campus Verlag, 1998.

Le Bihan, J., Au service de l'État. Les fonctionnaires intermédiaires au XIXe siècle, Rennes, PUR, 2008.

Liang, H. H., The Rise of Modern Police and the European State from Metternich to the Second World War, Cambridge, CUP, 1992.

Lignereux, A., La France rébellionnaire. Les résistances à la gendarmerie, 1800-1859, Rennes, PUR, 2008.

Lunn, J., Les races guerrières. Racial preconceptions in the French military about West African soldiers during the First World War, Journal of Contemporary History, 1999, 4, pp. 517-536.

Mbembe, A., De la postcolonie, essai sur l'imagination politique dans l'Afrique contemporaine, Paris, Karthala, 2000.

Memmi, Al, Portrait du colonisé. Portrait du colonisateur, Paris, Gallimard, 1985 (1 ère édition, 1957).

Mencherini, R., Guerre froide, grèves rouges. Parti communiste, stalinisme et luttes sociales en France. Les grèves «insurrectionnelles» de 1947-1948, Paris, Syllepse, 1998.

Merriman, J., Police Stories. Building the French State 1815-1851, Oxford, OUP, 2006.

Moreau de Bellaing, C., Comment la violence vient aux policiers? École de police et enseignement de la violence légitime, Genèses. Sciences sociales et histoire, 2009, 75, pp. 2444.

Morlang, T., Askari und Fitafita. «Farbige» Söldner in den deutschen Kolonien, Berlin, Christoph Links Verlag, 2008.

Milliot, V., Histoire des polices: l'ouverture d'un moment historiographique, Revue d'histoire moderne et contemporaine, 2007, 54, 2, pp. 162-177.

Milliot, V., Mais que font les historiens de la police? in Berlière, J.-M., Denys, C., Kalifa, D., Métiers de police. Etre policier en Europe, XVIII ${ }^{e}-X X^{e}$ siècles, Rennes, Presses universitaires de Rennes, 2008, pp. 9-34.

Mudimbe, V., The Invention of Africa. Gnosis, Philosophy and the Order of Knowledge, London, 1988.

Nord, P., Introduction in Bermeo, N., Nord, P., Civil society before Democracy, Lesson from nineteenth Century Europe, Boston, Rowman and Littlefield, 2000, pp. xiii-xxxiii.

Oloukpona-Yinnon, A., La révolte des esclaves-mercenaires. Douala 1893, Bayreuth, African Studies, Series 10, 1987.

Parsons, T., Wakamba Warriors Are Soldiers of the Queen: The Evolution of the Kamba as a Martial Race, 1890-1970, Ethnohistory, 1999, 4, pp. 671-701.

Pesek, M., Koloniale Herrschaft in Deutsch-Ostafrika. Expeditionen, Militär und Verwaltung seit 1880, Frankfurt, Campus, 2006.

Peyroulou, J.-P., Guelma 1945: une subversion européenne dans l'Algérie coloniale, Paris, La Découverte, 2009. 
Pruvost, G., Profession: policier. Sexe: féminin, Paris, éd. de la Maison des sciences de l'homme, 2007.

Ranger, T., The invention of Tradition in Colonial Africa, in Hobsbawm, E., Ranger, T. (Eds), The Invention of Tradition, Cambridge, CUP, 1983, pp. 211-262.

Ranger, T., The invention of tradition revisited: the case of colonial Africa, in Ranger, T., Vaughan, O., Legitimacy and The State in the Twentieth Century Africa: Essays in Honour of A.H.M. Kirk-Greene, Basingstoke, Macmillan, 1993, pp. 62-111.

Rotimi, K., The Police in a federal state. The Nigerian experience, Ibadan, College Press, 2001.

Schmitt, C., La notion de politique. Théorie du partisan, Paris, Flammarion, 1992 (1 ${ }^{\mathrm{èr}}$ éd. 1962).

Sinclair, G., The Irish policeman and the Empire, influencing the policing of the British Empire Commonwealth, Irish Historical Studies, 2008, 142, pp. 173-187.

Sinclair, G., Williams, C., 'Home and Away': The Cross-fertilisation between 'Colonial'and 'British'Policing, Journal of Imperial and Commonwealth History, 2007, 35, pp. 221238.

Singha, R., Settle, Mobilize, Verify: Identification Practices in Colonial India, Studies in History, 2000, 16, 2, pp.151-198.

Shuval, T., La ville d'Alger vers la fin du XVIII siècle. Population et cadre urbain, Paris, CNRS éditions, 1999.

Streets, H., Martial Races. The military, race and masculinity in British imperial culture, 1857-1914, Manchester, MUP, 2004.

Thioub, I., Sénégal: la prison à l'époque coloniale. Significations, évitements et évasions, in Bernault, F. (dir.), Enfermement, prison et châtiments en Afrique. Du XIX siècle à nos jours, Paris, Karthala, 1999, pp. 285-304.

Thomas, M., Empire of intelligence. Security Services and Colonial Disorder after 1914, Berkeley, University of California Press, 2008.

Trotha, T. von, Koloniale Herrschaft. Zur soziologischen Theorie der Staatsentstehung am Beispiel des «Schutzgebietes Togo», Tübingen, Mohr, 1994.

Verma, A., Cultural roots of police corruption in India. Policing, An International Journal of Police Strategies \& Management, 1999, 22, 3, pp. 264-279.

Vidal-Naquet, P., Les assassins de la mémoire, Paris, La Découverte, 1987.

Waddington, P., Controlling protest in contemporary historical and comparative perspective, in Della Porta, D., Reiter, H., Policing protest: The control of mass demonstrations in western democracies, Minneapolis, University of Minnesota Press, 1998, pp. 117-142.

Waddington, P., Wright, M., Police use of force, firearms and riot-control, in Newburn, T., Handbook of Policing, Cullompton, Willan Publishing, 2009 ( $2^{\text {nd }}$ ed.), pp. 465-493.

Wiener, M., An Empire on Trial: Race, Murder, and Justice under British Rule, 1970-1935, Cambridge, Cambridge University Press, 2008. 